\title{
Effect of docosahexaenoic acid on traumatic brain injury in rats
}

\author{
WEI ZHU*, NAN CHI", PENG ZOU, HONGGUANG CHEN, GUOTAI TANG and WEI ZHAO \\ Department of Neurosurgery, The Affiliated Yantai Yuhuangding Hospital of Qingdao University, \\ Yantai, Shandong 264000, P.R. China
}

Received September 7, 2016; Accepted June 20, 2017

DOI: 10.3892/etm.2017.5054

\begin{abstract}
The present study aimed to investigate the protective effects of docosahexaenoic acid (DHA) on traumatic brain injury (TBI) in rats. A model of TBI was induced by lateral fluid percussion injury in adult rats and rats were randomly divided into the TBI-model group, TBI-low DHA group and TBI-high DHA group, while other healthy rats were assigned to the sham-operated group. Motor recovery was tested with beam-walking trials at 2, 7 and 15 days post-TBI. Cognitive recovery was tested with Morris water maze trials at 15 days post-TBI. The expression levels of caspase-3, B-cell lymphoma 2 (Bcl-2) and Bcl-2-associated X protein (Bax) were measured by western blotting. DHA protected against motor deficits induced by TBI in beam walking tests. All TBI-model groups had longer escape latency and swimming distances than the sham groups. Compared with the TBI-low DHA group, the TBI-high DHA group demonstrated shorter escape latency and swimming distances. DHA inhibited the expression of caspase-3 and the inhibition effect was more obvious at a high dosage. Furthermore, DHA dose-dependently rescued neurons by upregulating the $\mathrm{Bcl}-2$ :Bax ratio. DHA supplementation was a viable strategy to mitigate injury from TBI.
\end{abstract}

\section{Introduction}

Traumatic brain injury (TBI) is usually caused by external mechanical force (1), such as falls, motor vehicle accidents, collisions, contact sports and assaults. Physical, behavioral, emotional and psychosocial changes may be observed in groups subjected to TBI (2). TBI is known to occur as the result of two phases: Initial neuronal injury followed by secondary injury. Initial neuronal injury occurs immediately and is due to the inciting traumatic event, which is not easily

Correspondence to: Dr Wei Zhu, Department of Neurosurgery, The Affiliated Yantai Yuhuangding Hospital of Qingdao University, 20 Yuhuangding East Road, Yantai, Shandong 264000, P.R. China E-mail: zhuwei20162016@126.com

*Contributed equally

Key words: B-cell lymphoma 2, B-cell lymphoma 2-associated X protein, caspase- 3 , docosahexaenoic acid, traumatic brain injury treatable. However, the second phase occurs from multiple neuropathological processes and evolves over a period of min to days (3-5). The delayed nature of secondary injuries allows for medical and surgical intervention and has become a major focus of TBI treatment (6). The secondary injuries are multiple, interacting and interdependent cascades of biological reactions caused by the initial injury (1). A series of complex biochemical process associated with the secondary injury occur after a traumatic brain injury $(7,8)$. In this phase, astrocyte foot process swelling may result in the damage of the blood-brain barrier (9). The injuries to the central nervous system, such as the proliferation of astrocytes, may also be detected, which may result in a reversal of glutamate uptake and neuronal depolarization $(10,11)$.

Polyunsaturated fatty acids (PUFAs) are able to maintain $\mathrm{Ca}^{2+}$ ion and energy homeostasis (12), decrease cognitive deficits and enhance learning ability during aging (13), and improve the prognosis of ischemic injury, Alzheimer's disease and Parkinson's disease (14). Docosahexaenoic acid (DHA) is a kind of polyunsaturated fatty acid, which is predominantly extracted from deep-sea fishes and algae (15). DHA is not only an important polyunsaturated fatty acid in the central nervous system, but it is also the main constituent of n-3 polyunsaturated fatty acids in cell membranes of cortical gray matter neurons (16). There is limited research on the impact of supplementation with PUFAs on TBI (17-19). Nevertheless, these studies (17-19) have consistently demonstrated the protective effects of PUFAs against behavioral deficits and cellular degeneration.

DHA has a negative effect on damaging factor production, such as inflammatory cytokines and free radicals $(20,21)$. The present study characterized the impact of DHA supplementation on cognitive function and inflammatory responses following fluid percussion injury (FPI) in rats.

\section{Materials and methods}

Animal groups. A total of 807 -week old Sprague-Dawley rats (male/female ratio 1:1) weighing 300-500 g were maintained in a temperature $\left(21-25^{\circ} \mathrm{C}\right)$-and humidity $(45-50 \%)$-controlled room with a 12-h light/dark cycle with ad libitum access to food and water. Following 1 week of acclimation, the rats were randomly divided into four groups (male/female ratio 1:1): i) A TBI-model group (post-TBI with saline; $\mathrm{n}=20$ ); ii) a sham-operated group treated with saline $(n=20)$; iii) a TBI-low DHA group, treated with a low dose of DHA post-TBI ( $\mathrm{n}=20 ; 370 \mathrm{mg} / \mathrm{kg} /$ day DHA); 
and iv) a TBI-high DHA group, treated with a high dose of DHA post-TBI ( $\mathrm{n}=20 ; 740 \mathrm{mg} / \mathrm{kg} / \mathrm{day}$ DHA). The present study was approved by the Ethics Committee of Yantai Yuhuangding Hospital (Yantai, China).

Animal models of TBI. Rats were anesthetized by injection of $10 \%$ chloral hydrate $(300 \mathrm{mg} / \mathrm{kg}$; Sinopharm Chemical Reagent Co., Ltd., Shanghai, China), administered intraperitoneally. A $4.5-\mathrm{mm}$ diameter window in the bone was made $3.5-\mathrm{mm}$ posterior to the bregma and $2.5-\mathrm{mm}$ lateral to the sagittal suture, without injuring the dura mater. The head of the rat was positioned in a stereotaxic alignment instrument for FPI. For FPI, rats were connected through a craniotomy to a fluid-filled chamber with a small opening; a swinging pendulum hit one end of the chamber to generate a water pulse that impacted the exposed brain at the other end of the chamber. Depending on the location of the craniotomy, the injury could be delivered to the side of the brain (lateral FPI) or the midline (central FPI), and the craniotomy was performed at the same location in all of the rats to the midline. Injury intensity was controlled by adjusting the height from which the pendulum was dropped (22). Sham-operated rats underwent craniotomy without FPI (23).

DHA application. Following TBI injury, rats were randomized to three groups: TBI-model group, TBI-low DHA group, TBI-high DHA group ( $\mathrm{n}=20$ per group). DHA (Sigma-Aldrich; Merck KGaA; Darmstadt, Germany) was given by intragastric administration. The sham and TBI model group received equal volumes of saline treatment $(0.9 \% \mathrm{NaCl} ; 1 \mathrm{ml} / \mathrm{kg})$. Treatment administration began 30 min after TBI injury and continued once a day for 15 days.

Beam-walking tests. To evaluate complex motor movements and coordination, beam-walking tests were performed 1 day prior to TBI and on days 2, 7 and 15 post-TBI (24). The beam was a wooden bar $1,390 \mathrm{~mm}$ in length and $21 \mathrm{~mm}$ wide, and was placed $430 \mathrm{~mm}$ above the floor. There was a black box $(250 \times 200 \mathrm{~mm})$ at the right end of the beam. A wall was placed $30 \mathrm{~cm}$ to the left of the beam, as rats are more willing to walk when a wall is placed next to the beam. A mirror was behind the beam on the side wall. Starting at 2 days before TBI, rats were habituated to walk on the beam. A rat was put into the box for $1 \mathrm{~min}$. Then, the rat was put onto the beam at a starting distance of $15 \mathrm{~cm}$ from the box. The rat was allowed to go to the box and stay there for $1 \mathrm{~min}$. Thereafter, the rat was put on the beam at a starting distance of $35 \mathrm{~cm}$ from the box. The rat was allowed to go into the box (and stayed for $1 \mathrm{~min}$ ). This step was repeated. On the following day, the rat was put into the box for $1 \mathrm{~min}$ and then allowed to go to the box starting from $35 \mathrm{~cm}$, followed by $70 \mathrm{~cm}$ and finally from a $100-\mathrm{cm}$ distance from the box. On the testing day, the rat was allowed to cross the whole beam three times. Between each run, the rat was in the box for $1 \mathrm{~min}$. Scoring was as follows: $0=$ the rat fell down; $1=$ the rat was unable to traverse the beam but remained sitting across the beam; $2=$ the rat fell down during its walk; $3=$ the rat was able to traverse the beam, but the affected hindlimb did not aid in forward locomotion; $4=$ the rat traversed the beam with $>3$ foot slips; $5=$ the rat crossed the beam with 1-3 foot slips; and $6=$ the rat crossed

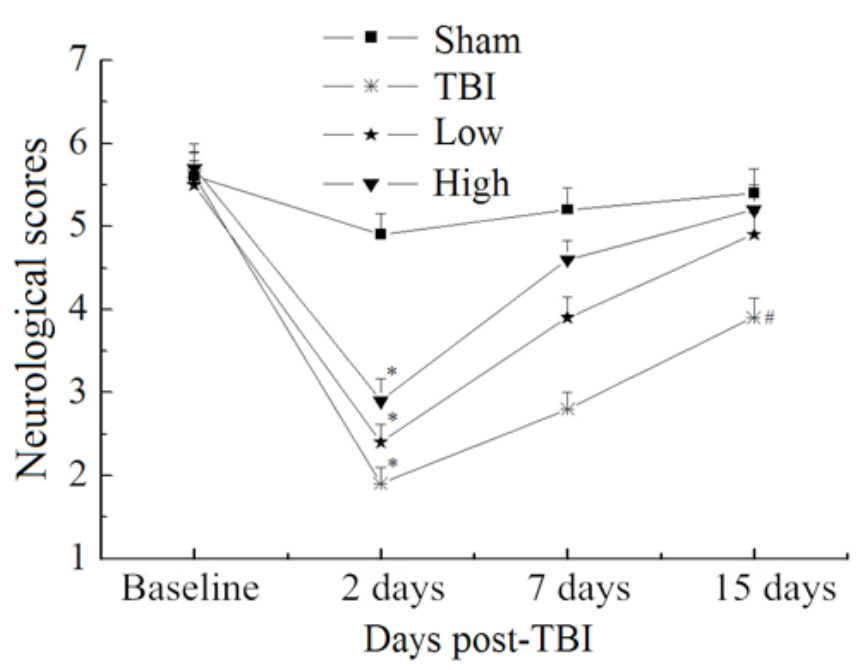

Figure 1. Performance scores for beam-walking tests. Analysis of performance using $\mathrm{R}$ software revealed differences between the groups. At 2 days post-TBI, the TBI, low and high groups were significantly impaired compared with the sham group. Both treatment groups recovered quickly during the first week post-TBI. At 15 days post-TBI, performance in groups treated with DHA did not significantly differ from that in the sham group, whereas the TBI model group remained significantly more impaired compared with the sham group. ${ }^{*} \mathrm{P}<0.05$ vs. the sham group at 2 days; ${ }^{\#} \mathrm{P}<0.05$ vs. the sham group at 15 days. TBI, traumatic brain injury; DHA, docosahexaenoic acid; low, low-dose DHA (370 mg/kg/day); high, high-dose DHA (740 mg/kg/day).

the beam with no foot slips. A mean score of the three runs for each day was calculated.

Morris water maze trials. The water maze test was initiated at day 15 after the induction of TBI. The rat was placed on the platform submerged below the water for $20 \mathrm{sec}$ to allow orientation to extra-maze cues. The rat was then placed in the water tank at one of four designated entry points (west, north, east and south) facing the wall and the time taken to reach the hidden platform was recorded for each trial. In addition, swimming speed and path length (swimming distance) were measured. A probe test was conducted 15 days after TBI, during which the platform was removed. Rats were allowed to swim for $60 \mathrm{sec}$ to allow evaluation of their memory of the platform location. The time spent in the four quadrants of the maze was recorded. A total of eight trials per day were averaged for each rat and the mean score was calculated for swimming on days 16 and 17 post-TBI. The tracks from all tests were analyzed for a series of behavioral parameters using SMART 3.0 (Panlab; Barcelona, Spain).

Histopathological evaluation. Brain specimens were collected and fixed in $10 \%$ formalin for $24 \mathrm{~h}$ at room temperature, embedded in paraffin. Sections of 4-mm thickness were cut from formalin-fixed tissues and stained with hematoxylin and eosin (10 min for hematoxylin staining and $5 \mathrm{~min}$ eosin staining at room temperature). Specimens were examined under a light microscope (magnification, x200).

Western blot analysis. Rats were deeply anesthetized with isoflurane and perfused transcardially with saline following administration of DHA for 15 days. The injured brain tissues were collected and homogenized in radioimmunoprecipitation 

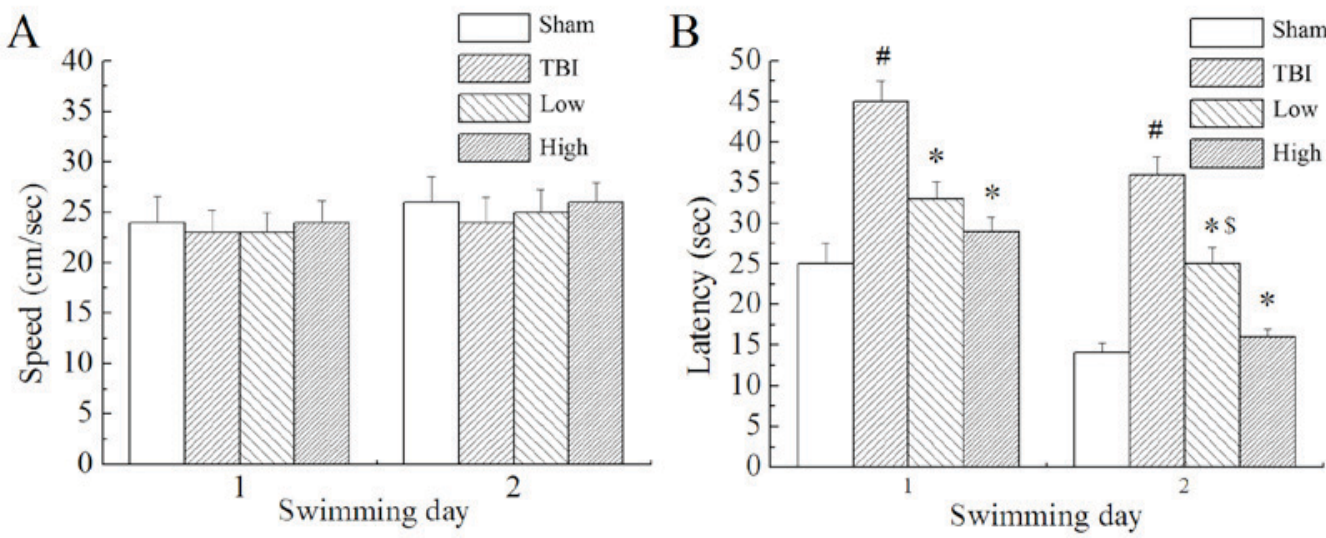

Figure 2. Performance in Morris water maze testing. (A) Swimming speeds in the Morris water maze trials were not significantly different between the groups. (B) Latency to find the hidden platform in the Morris water maze trials was significantly increased in the TBI model group compared with the sham group, while the groups that received treatment with DHA were not significantly different compared with the sham group. ${ }^{*} \mathrm{P}<0.05$ vs. the sham group; ${ }^{*} \mathrm{P}<0.05$ vs. the TBI group. ${ }^{\$} \mathrm{P}<0.05$ vs. the TBI-high DHA group TBI, traumatic brain injury; DHA, docosahexaenoic acid; low, low-dose DHA ( $\left.370 \mathrm{mg} / \mathrm{kg} / \mathrm{day}\right) ; \mathrm{high}$, high-dose DHA $(740 \mathrm{mg} / \mathrm{kg} / \mathrm{day})$.

assay buffer (89900; Thermo Scientific, Inc., Waltham, MA, USA) containing protease inhibitor cocktail (P2714) and protease inhibitor mixture (P2714; both Sigma-Aldrich; Merck KGaA, Darmstadt, Germany). Homogenates were centrifuged at $13,000 \mathrm{xg}$ at $4^{\circ} \mathrm{C}$ for $30 \mathrm{~min}$. The supernatant was saved to determine its protein concentration by Bradford assay. Total protein $(50 \mu \mathrm{g} / \mathrm{lane})$ was separated by $10 \%$ SDS-PAGE and then blotted onto a polyvinylidene difluoride membrane. Following blocking ( $2 \mathrm{~h}$ at room temperature) with ProteinFree T20 Blocking Buffer (37573; lot no. LB141635; Thermo Scientific, Inc.), membranes were incubated for $1 \mathrm{~h}$ at room temperature with the following primary antibodies: Rabbit monoclonal anti-cleaved caspase-3 antibody (1:1,000; 9664; Cell Signaling Technology, Inc., Danvers, MA, USA), rabbit anti-B-cell lymphoma 2 (Bcl-2; 1:1,000; 2872; Abcam, Cambridge, MA, USA), rabbit anti-Bcl-2-associated $\mathrm{X}$ protein (Bax; 1:1,000; 2772; Abcam) and rat monoclonal anti- $\beta$-actin polyclonal antibody (1:2,000; A2228; Sigma-Aldrich; Merck $\mathrm{KGaA})$. The membranes were subsequently incubated with horseradish peroxidase-conjugated secondary antibody at room temperature for $40 \mathrm{~min}(1: 5,000$; goat anti-rabbit, ZB-2301; goat anti-mouse, ZDR5307; ZSGB-BIO Technology Co., Ltd., Beijing, China). Protein bands were visualized using an enhanced chemiluminescence reagent (EMD Millipore; Billerica, MA, USA) and quantified by densitometry using a Genomic and Proteomic Gel Documentation System from Syngene (Frederick, MD, USA). The protein band intensities of Bcl-2, Bax and caspase-3 were normalized by the corresponding band intensities of $\beta$-actin from the same samples to control for loading errors. The results from animals under various experimental conditions were then normalized by mean values of the corresponding control animals.

Statistical analysis. Statistical analysis was performed using SPSS 19.0 software (IBM Corp., Armonk, NY, USA) for Windows. All data were presented as the mean \pm standard deviation. The significance of differences between groups was evaluated using one-way analysis of variance followed by
Dunnett's test. $\mathrm{P}<0.05$ was considered to indicate a statistically significant difference.

\section{Results}

DHA protects against motor deficits induced by TBI. Beam-walking trials were performed to evaluate complex motor movements and coordination. The results are summarized in Fig. 1. Further analysis at each time point did not reveal any significant differences in beam-walking between the groups at baseline $(\mathrm{P}>0.05)$. All groups were significantly impaired at 2 days post-TBI compared with the sham group $(\mathrm{P}<0.05)$. Both the TBI-low and TBI-high DHA groups improved over the 15-day follow-up. The changes observed over time in the TBI-model group were less notable. At 15 days post-TBI, the performance of the TBI-high and -low DHA groups approached that of the sham group $(\mathrm{P}>0.05)$; however, the neurological scores of the TBI model and TBI-low DHA groups were significantly lower than those of the sham group $(\mathrm{P}<0.05)$.

Morris water maze trials. In the Morris water maze testing, the rats were made to find the target platform to escape from swimming in the pool with water. Results demonstrated that there was no significant difference in swimming speed between the groups (Fig. 2A). However, there were significant differences in escape latency (time taken to find the submerged platform; $\mathrm{P}<0.05$; Fig. $2 \mathrm{~B}$ ) and marked differences in swimming distance (not shown) between the groups. The TBI model group demonstrated significantly longer escape latencies and swimming distances than the sham group $(\mathrm{P}<0.05)$. Compared with the TBI model group, the TBI-low and TBI-high DHA groups demonstrated significantly shorter latency times $(\mathrm{P}<0.05)$. Compared with the TBI-low DHA group, the TBI-high DHA group demonstrated shorter escape latencies and swimming distances.

Pathological findings of brain tissue. In the sham group, the majority of cells in the brain tissue demonstrated normal 

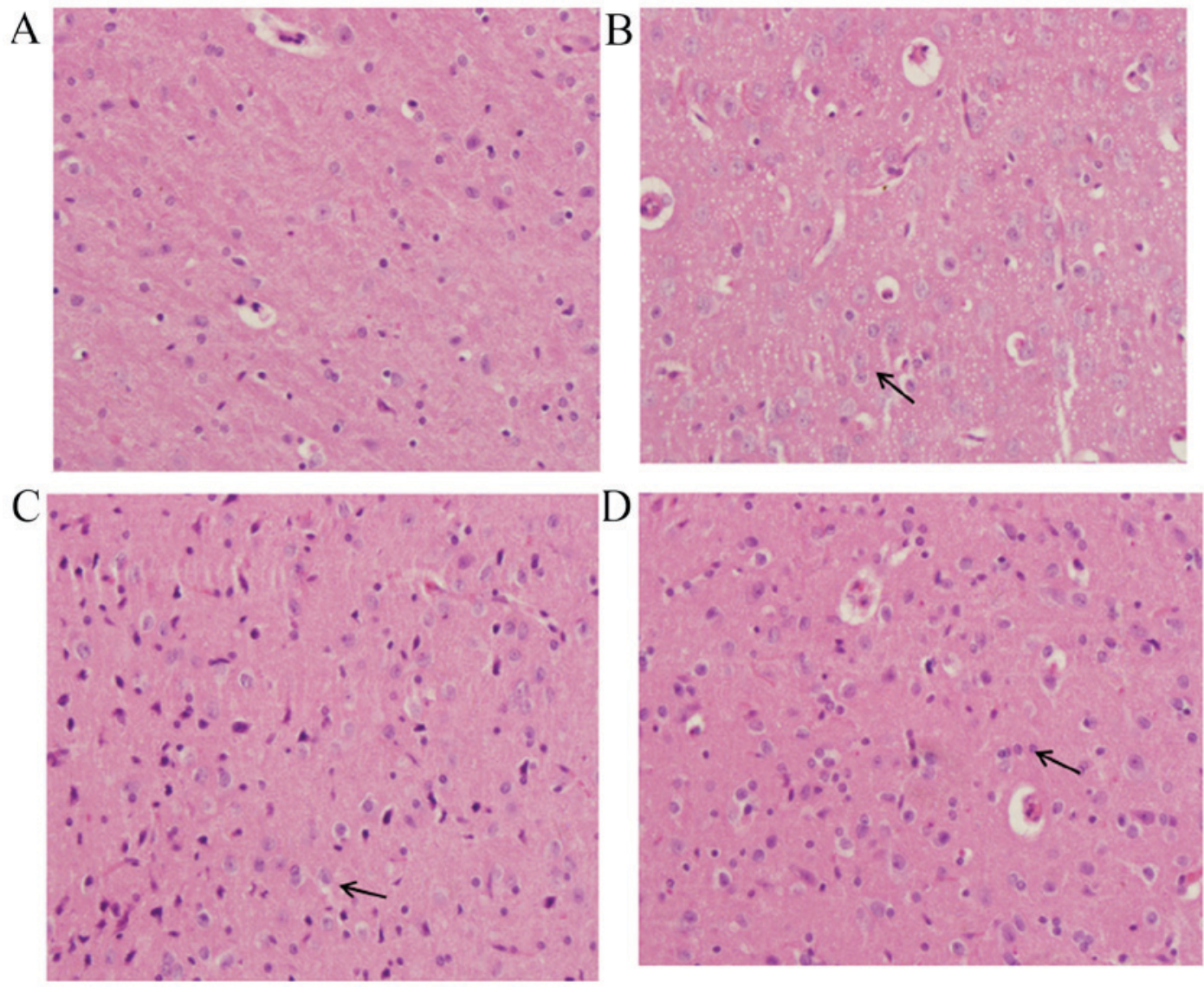

Figure 3. Pathological changes to brain tissue. Brain tissue specimens (stain, hematoxylin and eosin; magnification, x200) of the (A) sham-operated group, (B) TBI model group, (C) TBI group treated with a high dose of DHA and (D) TBI group treated with a low dose of DHA. TBI, traumatic brain injury; DHA, docosahexaenoic acid. Arrows indicated damaged glial cells revealing swelling status which were attenuated by DHA treatment.

morphology (Fig. 3A). Evidently damaged nerve cell structure, swelling of nerve cells, shrinking of nucleolus, obvious hyperemia and congestion in blood capillaries, the proliferation of glial cells and the formation of clusters of neurons and the 'satellite phenomenon' in glial cells were observed in the TBI-model group (Fig. 3B). The changes observed in the DHA-treated groups were milder than those of the TBI-model group, which appeared to be dose-dependent. The group treated with the high dose of DHA demonstrated basically normal structure of brain tissue, slight hyperemia and congestion in blood capillaries and the proliferation of glial cells (Fig. 3C). While the group treated with the low dose of DHA (Fig. 3D) demonstrated obvious pathological changes compared with the high DHA-treated group.

DHA inhibits the expression of caspase-3. Caspase-3 was constitutively expressed in the sham-operated group, while the TBI-model group (post-TBI with saline) demonstrated a significant increase in the expression of caspase- 3 compared with the sham group $(\mathrm{P}<0.05)$. When treated with DHA, the expression of caspase- 3 was significantly reduced compared with that in the rats of the TBI model groups $(\mathrm{P}<0.05)$, indicating that DHA inhibited the expression of caspase-3 (Fig. 4). The inhibitory effect of DHA was more obvious at a high dosage.

DHA rescues neurons by upregulating the Bcl-2:Bax ratio. In the rats of the sham group, the expression level of Bcl-2 and Bax was significantly lower than those in the other groups $(\mathrm{P}<0.05)$. The results demonstrated significant upregulation of $\mathrm{Bcl}-2$ and downregulation of Bax in the DHA groups compared with the TBI-model group ( $\mathrm{P}<0.05$; Fig. 5).

\section{Discussion}

Traumatic brain injury is a prevalent neurological disorder that results in gray and white matter injury (25). In the present study, 15 days of supplementation with DHA elicited robust protection against sensorimotor and cognitive deficits in a rat model of TBI. This protective dietary strategy has previously demonstrated much success in other paradigms $(26,27)$. In the beam-walking test, further analysis at each time point did not reveal any significant differences in beam-walking between the groups at baseline, which suggested that learning ability was maintained in the injured group. All groups were impaired significantly on day 2 post-TBI compared with the sham group, followed by a gradual increase thereafter. However, a significant increase in score was observed in rats that received DHA relative to the TBI-model rats, suggesting that the administration of DHA significantly improved the motor movements and coordination of rats. Analogously, the rats that received treatment with DHA performed better than TBI-model rats in Morris water maze trials. All TBI-related groups demonstrated longer escape latencies and swimming distances than the sham group. Compared with the TBI-low DHA group, the 
A
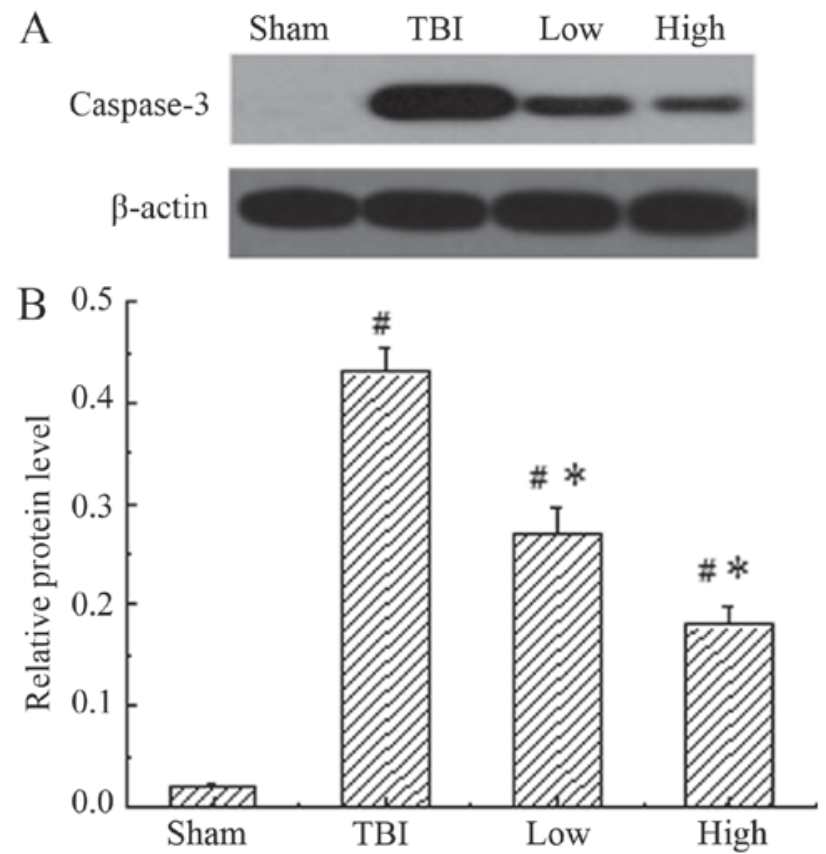

Figure 4. Western blot analysis of the protein expression levels of caspase-3. (A) Representative western blot images and (B) quantification of caspase-3 protein expression levels. Low expression of caspase-3 was demonstrated in the rats of the sham group, while the TBI-model rats (post-TBI with saline) demonstrated a significant increase in the expression of caspase-3 compared with the sham group. When treated with DHA, the expression of caspase-3 was significantly reduced compared with that in rats of the TBI model group. ${ }^{~} \mathrm{P}<0.05$ vs. the sham group; ${ }^{*} \mathrm{P}<0.05$ vs. the TBI model group. TBI, traumatic brain injury; DHA, docosahexaenoic acid; low, low-dose DHA (370 mg/kg/day); high, high-dose DHA (740 mg/kg/day).

TBI-high DHA group had shorter escape latency and swimming distances. According to a study by Bailes and Mills (28), supplementation with DHA significantly decreased amyloid precursor protein-positive axons in the white matter tract.

In models of TBI, pro-apoptotic mechanisms may be activated during secondary damage to promote caspase-3-mediated cell death (29). In the present study, it was demonstrated that the expression of active caspase-3 was downregulated in the cortex of rats treated with DHA following TBI, which protected the cortical neurons from apoptosis. Caspase- 3 activation in cell death signaling may occur via three routes: The mitochondrial route (related to $\mathrm{Bcl}-2$ and $\mathrm{Bax}$ ), the endoplasmic reticulum route or a death receptor route involving FAS and FAS ligand (30). Bcl-2 gene families have been identified to be regulators of apoptosis. Of these genes, Bcl-2 is an anti-apoptotic protein that serves as a critical regulator of pathways involved in apoptosis. Contrastingly, Bax is a pro-apoptotic protein, which controls the integrity of the mitochondrial outer membrane (31). Research has indicated that the $\mathrm{Bcl}-2$ protein physically interacts with several of its homologous proteins, forming heterotypic dimers (32). The $\mathrm{Bcl}-2 / \mathrm{Bax}$ dimerization is considered critical for interactions during apoptosis (33). Bcl-2 is downregulated in the injured brain (34) and the overexpression of $\mathrm{Bcl}-2$ reduces post-ischemic injury (35). Chronic daily administration of DHA significantly increased Bcl-2 expression in brain tissues in the present study.
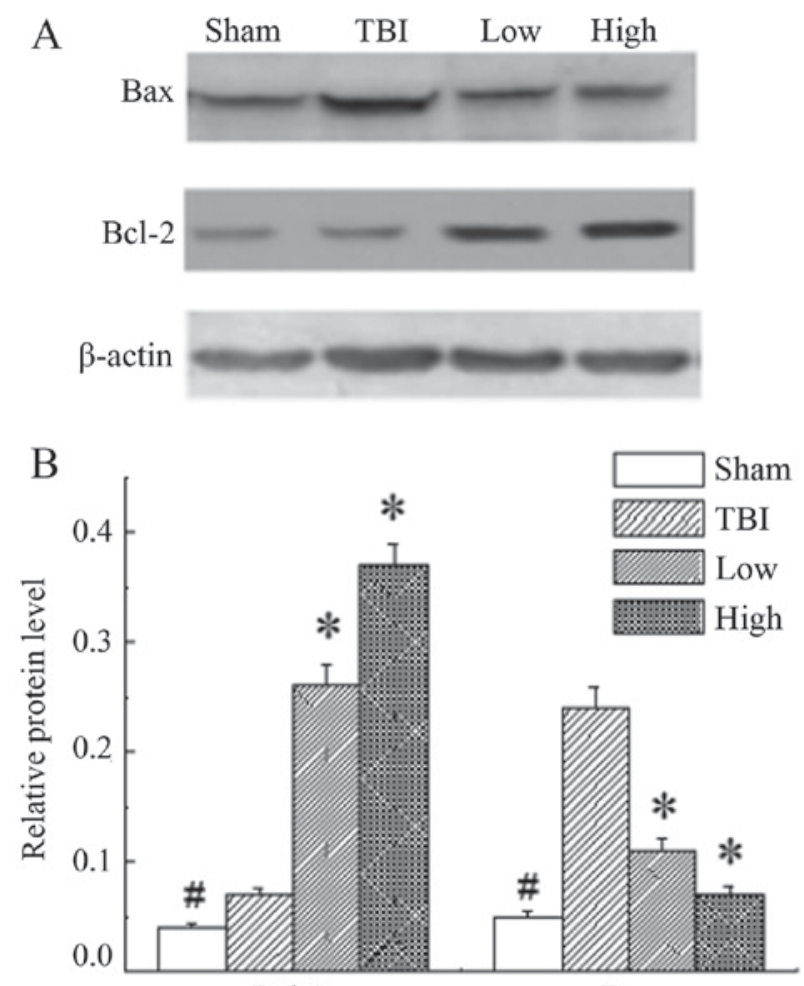

Bcl-2

$\mathrm{Bax}$

Figure 5. Bcl-2 and Bax protein expression levels in the different groups. (A) Representative western blot images of Bcl-2 and Bax protein expression levels. (B) Graphic presentation of $\mathrm{Bcl}-2$ and Bax protein expression levels. The expression levels of $\mathrm{Bcl}-2$ and $\mathrm{Bax}$ in the sham group were significantly lower than those of the other groups. The results demonstrated significant upregulation of Bcl-2 and downregulation of Bax in the groups treated with DHA compared with the TBI model group. ${ }^{*} \mathrm{P}<0.05$ vs. all other groups; "P<0.05 vs. the TBI model group. Bcl-2, B-cell lymphoma 2; Bax, B-cell lymphoma 2-assocaited X protein; TBI, traumatic brain injury; DHA, docosahexaenoic acid; low, low-dose DHA (370 mg/kg/day); high, high-dose DHA $(740 \mathrm{mg} / \mathrm{kg} /$ day).

To determine the signal that modulated the activation of caspase- 3 following TBI, the present study measured the protein expression levels of Bcl-2 and Bax in order to calculate the Bcl-2:Bax ratio. The results demonstrated the upregulation of Bcl-2 and the downregulation of Bax protein levels following treatment with DHA. Therefore, these results indicated that DHA may increase the Bcl-2:Bax ratio to inhibit the expression of caspase- 3 , ultimately protecting neurons from apoptosis.

In conclusion, the present study demonstrated that DHA supplementation was a viable strategy to mitigate injury caused by TBI. DHA treatment improved memory function, which may be due to its anti-inflammatory properties. Furthermore, DHA was useful in preventing neuronal damage following brain ischemia. The present data support the belief that fish oil supplementation in humans may exert similar prophylactic or preventive actions against neuronal damage in the future.

\section{References}

1. Maas AI, Stocchetti N and Bullock R: Moderate and severe traumatic brain injury in adults. Lancet Neurol 7: 728-741, 2008. 
2. Lauterbach MD, Notarangelo PL, Nichols SJ, Lane KS and Koliatsos VE: Diagnostic and treatment challenges in traumatic brain injury patients with severe neuropsychiatric symptoms: Insights into psychiatric practice. Neuropsychiatr Dis Treat 11: $1601-1607,2015$.

3. McIntosh TK: Neurochemical sequelae of traumatic brain injury: Therapeutic implication. Cerebrovasc Brain Metabol Rev 6: 109-162, 1994.

4. Park E, Bell JD and Baker AJ: Traumatic brain injury: Can the consequences be stopped. CMAJ 178: 1163-1170, 2008.

5. Raghupathi R, Conti AC, Graham DI, Krajewski S, Reed JC, Grady MS, Trojanowski JQ and McIntosh TK: Mild traumatic brain injury induces apoptotic cell death in the cortex that is preceded by decreased in cellular Bcl-2 immunoreactivity. Neuroscience 110: 605-616, 2002.

6. McHugh GS, Engel DC, Butcher I, Steyerberg EW, Lu J, Mushkudiani N, Hernández AV, Marmarou A, Maas AI and Murray GD: Prognostic value of secondary insults in traumatic brain injury: Results from the IMPACT study. J Neurotrauma 24: 287-293, 2007

7. King JT Jr, Carlier PM and Marion DW: Early glasgow outcome scale scores predict long-term functional outcome in patients with severe traumatic brain injury. J Neurotrauma 22: 947-954, 2005.

8. Fork M, Bartels C, Ebert AD, Grubich C, Synowitz H and Wallesch CW: Neuropsychological sequelae of diffuse traumatic brain injury. Brain Inj 19: 101-108, 2005.

9. Bao HJ, Wang T, Zhang MY, Liu R, Dai DK, Wang YQ, Wang L, Zhang L, Gao YZ, Qin ZH, et al: Poloxamer-188 attenuates TBI-induced blood-brain barrier damage leading to decreased brain edema and reduced cellular death. Neurochem Res 37 2856-2867, 2012

10. Lenzlinger PM, Morganti-Kossmann MC, Laurer HL and McIntosh TK: The duality of the inflammatory response to traumatic brain injury. Mol Neurobiol 24: 169-181, 2001

11. Liou AK, Clark RS, Henshall DC, Yin XM and Chen J: To die or not to die for neurons in ischemia, traumatic brain injury and epilepsy: A review on the stress-activated signaling pathways and apoptotic pathways. Prog Neurobiol 69: 103-142,2003.

12. Wu A, Ying Z and Gomez-Pinilla F: The salutary effects of DHA dietary supplementation on cognition, neuroplasticity and membrane homeostasis after brain trauma. J Neurotrauma 28 : 2113-2122, 2011.

13. Cole GM, Ma QL and Frautschy SA: Dietary fatty acids and the aging brain. Nutr Rev 68: S102-111, 2010.

14. Bousquet $M$, Calon $F$ and Cicchetti $F$ : Impact of $\omega-3$ fatty acids in Parkinson's disease. Ageing Res Rev 10: 453-463, 2011.

15. Kalmijn S, Feskens EJ, Launer LJ and Kromhout D Polyunsaturated fatty acids, antioxidants and cognitive function in very old men. Am J Epidemiol 145: 33-41, 1997.

16. Dyall SC: Long-chain omega-3 fatty acids and the brain: A review of the independent and shared effects of EPA, DPA and DHA. Front Aging Neurosci 7: 52, 2015.

17. Wu A, Ying Z and Gomez-Pinilla F: Omega-3 fatty acids supplementation restores mechanisms that maintain brain homeostasis in traumatic brain injury. J Neurotrauma 24: 1587-1595, 2007.

18. Chang PK, Khatchadourian A, McKinney RA and Maysinger D Docosahexaenoic acid (DHA): A modulator of microglia activity and dendritic spine morphology. J Neuroinflammation 12: 34 , 2015.

19. Pusceddu MM, Kelly P, Stanton C, Cryan JF and Dinan TG: N-3 polyunsaturated fatty acids through the lifespan: Implication for psychopathology. Int J Neuropsychopharmacol 19: pyw078, 2016.

20. Chen W, Esselman WJ, Jump DB and Busik JV: Anti-inflammatory effect of docosahexaenoic acid on cytokine-induced adhesion molecule expression in human retinal vascular endothelial cells. Invest Ophthalmol Vis Sci 46: 4342-4347, 2005.
21. Florent S, Malaplate-Armand C, Youssef I, Kriem B, Koziel V, Escanyé MC, Fifre A, Sponne I, Leininger-Muller B, Olivier JL, et al: Docosahexaenoic acid prevents neuronal apoptosis induced by soluble amyloid-beta oligomers. J Neurochem 96: 385-395, 2006.

22. Gyoneva S and Ransohoff RM: Inflammatory reaction after traumatic brain injury: Therapeutic potential of targeting cell-cell communication by chemokines. Trends Pharmacol Sci 36: 471-480, 2015.

23. McIntosh TK, Vink R, Noble L, Yamakami I, Fernvak S, Soares H and Faden AL: Traumatic brain injury in the rat: Characterization of a lateral fluid-percussion model. Neuroscience 28: 233-244, 1989.

24. Ohlsson AL and Johansson BB: Environment influences functional outcome of cerebral infarction in rats. Stroke 26: 644-649, 1995.

25. Gasparovic C, Yeo R, Mannell M, Ling J, Elgie R, Phillips J, Doezema D and Mayer AR: Neurometabolite concentrations in gray and white matter in mild traumatic brain injury: An $1 \mathrm{H}$-magnetic resonance spectroscopy study. J Neurotrauma 26 : 1635-1643, 2009.

26. Nguemeni C, Delplanque B, Rovère C, Simon-Rousseau N, Gandin C, Agnani G, Nahon JL, Heurteaux C and Blondeau N: Dietary supplementation of alpha-linolenic acid in an enriched rapeseed oil diet protects from stroke. Pharmacol Res 61: 226-233, 2010

27. Martín A, Boisgard R, Kassiou M, Dollé F and Tavitian B: Reduced PBR/TSPO expression after minocycline treatment in a rat model of focal cerebral ischemia: A PET study using (18)F] DPA-714. Mol Imaging Biol 13: 10-15, 2011.

28. Bailes JE and Mills JD: Docosahexaenoic acid reduces traumatic axonal injury in a rodent head injury model. J Neurotrauma 27: 1617-1624, 2010.

29. Clark RS, Kochanek PM, Watkins SC, Chen M, Dixon CE, Seidberg NA, Melick J, Loeffert JE, Nathaniel PD, Jin KL and Graham SH: Caspase-3 mediated neuronal death after traumatic brain injury in rats. J Neurochem 74: 740-753, 2000.

30. Salakou S, Kardamakis D, Tsamandas AC, Zolota V, Apostolakis E, Tzelepi V, Papathanasopoulos P, Bonikos DS, Papapetropoulos T, Petsas T and Dougenis D: Increased $\mathrm{Bax} / \mathrm{Bcl}-2$ ratio up-regulates caspase- 3 and increases apoptosis in the thymus of patients with myasthenia gravis. In Vivo 21: $123-132,2007$

31. Solaroglu I, Tsubokawa T, Cahill J and Zhang JH: Anti-apoptotic effect of granulocyte-colony stimulating factor after focal cerebral ischemia in the rat. Neuroscience 143: 965-974, 2006.

32. Hara A, Hirose Y, Wang A, Yoshimi N, Tanaka T and Mori $\mathrm{H}$ : Localization of $\mathrm{Bax}$ and $\mathrm{Bcl}-2$ proteins, regulators of programmed cell death, in the human central nervous system. Virchows Arch 429: 249-253, 1996.

33. Xiao D and Zhang L: Upregulation of Bax and Bcl-2 following prenatal cocaine exposure induces apoptosis in fetal rat brain. Int J Med Sci 5: 295-302, 2008.

34. Kao TK, Ou YC, Kuo JS, Chen WY, Liao SL, Wu CW, Chen CJ, Ling NN, Zhang YH and Peng WH: Neuroprotection by tetramethylpyrazine against ischemic brain injury in rats. Neurochem Int 48: 166-176, 2006

35. Wei L, Cui L, Snider BJ, Rivkin M, Yu SS, Lee CS, Adams LD, Gottlieb DI, Johnson EM Jr, Yu SP and Choi DW: Transplantation of embryonic stem cells overexpressing Bcl-2 promotes functional recovery after transient cerebral ischemia. Neurobiol Dis 19: 183-193, 2005. 